\title{
A gold-catalysed fully intermolecular oxidation and sulfur-ylide formation sequence on ynamides $\dagger$
}

50, 6001

Received 10th February 2014

Accepted 16th April 2014

DOI: $10.1039 / c 4 c c 01059 k$

www.rsc.org/chemcomm

\author{
Mickaël Dos Santos and Paul W. Davies*
}

An efficient $\mathrm{C}-\mathrm{O}, \mathrm{C}-\mathrm{S}$ and $\mathrm{C}-\mathrm{C}$ bond-forming sequence leads to functionalised compounds bearing sulfur-substituted quaternary carbons. Ynamides are employed as diazo-equivalents to access the $[2,3]$-sigmatropic rearrangements of allyl sulfonium ylides by a three-component chemoselective oxidation and intermolecular ylide formation.

The $[2,3]$-sigmatropic rearrangement of allyl sulfonium ylides is a potent method for the formation of $\mathrm{Csp}^{3}-\mathrm{Csp}^{3}$ bonds. ${ }^{1}$ A significant hydrocarbon functionalisation process is achieved when the rearrangement is coupled with in situ ylide formation through reaction of a sulfide with a metal carbene, formed in situ from a sacrificial functionality such as the diazo-group (Doyle-Kirmse reaction, Scheme 1). ${ }^{2,3} \mathrm{We}^{4}$ and others ${ }^{5}$ have been engaged in efforts to access the synthetic potential of these ylides using methods to generate carbenoids that avoid the pre-installation and use of potentially hazardous high-energy diazo groups. Our initial studies established that sulfur ylides can be prepared from the intermolecular reaction of sulfides with gold-carbenes (Scheme 2). ${ }^{4 a, d, 6}$ However, the use of propargylic carboxylates as carbenoid precursors impacted on the subsequent ylidic rearrangements to generally afford products isomeric to those from [2,3]-sigmatropic pathways. More-congested centres and synthetically valuable sulfur-substituted quaternary carbons were inaccessible as terminal alkynes and unsubstituted allyl groups on the sulfide ${ }^{7}$ were required. ${ }^{4 a, d}$ We subsequently established that sigmatropic-rearrangements of sulfur ylides were accessible through an intramolecular gold or platinum catalysed cycloisomerisation of alkynyl allyl sulfoxides. ${ }^{4 b, 8}$ Here we report a diazo-free oxidation-ylide formation sequence to access sulfur-ylide rearrangements by a selective and efficient fully intermolecular transformation of ynamides.

In light of our, ${ }^{9}$ and others ${ }^{10}$ studies into gold-catalysed intermolecular atom/group-transfer onto ynamides $\mathbf{A},{ }^{11}$ we questioned

School of Chemistry, University of Birmingham, Edgbaston, B15 2TT, Birmingham, UK. E-mail: p.w.davies@bham.ac.uk; Tel: +44 (0)121 4144408

$\dagger$ Electronic supplementary information (ESI) available: Synthetic procedures and characterisation for all compounds. CCDC 984457. For ESI and crystallographic data in CIF or other electronic format see DOI: 10.1039/c4cc01059k

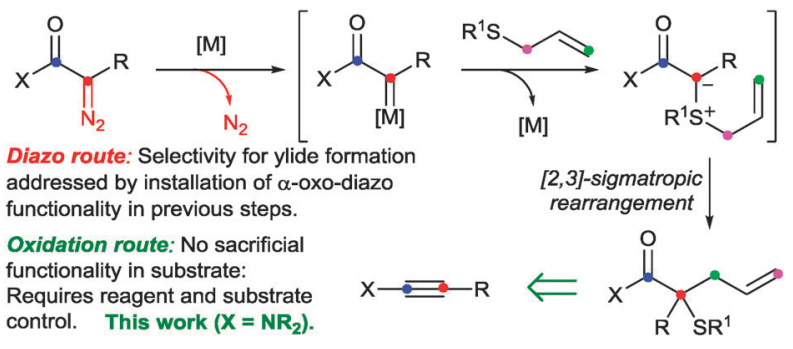

Scheme 1 The Doyle-Kirmse reaction and an alternate oxidation based route.

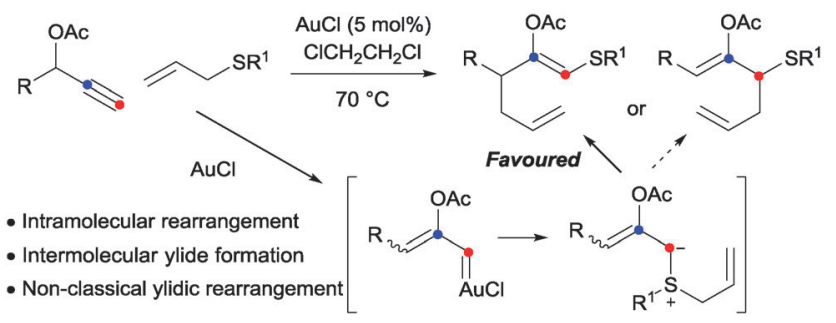

Scheme 2 Intermolecular trapping of gold carbenes with allyl sulfides: ref. $4 a, d$

whether ynamides could be used to replace diazo compounds in intermolecular sulfonium ylide formation to form quaternary carbons (Schemes 1 and 3).

Significant transformations have resulted from $\alpha$-oxo-gold carbene formation by intermolecular oxidation of a C-C triple bond. ${ }^{9,10,12}$ While these predominantly feature subsequent intramolecular cyclisations, there are striking exceptions in the terminal alkyne series where the mono-substituted gold carbene is trapped with intermolecular oxygen-, nitrogen- and halide nucleophiles other than the oxidant. ${ }^{13}$ Similar three-component couplings have not however been reported in the ynamide series and it is notable that the resulting 1,1-disubstituted organogold species $\mathbf{C / D}$ appears prone to oxidation even when intramolecular pathways are available. ${ }^{9,10,14}$ A successful outcome therefore requires a high level of reagent-compatibility and selectivity: the sulfide must not affect 

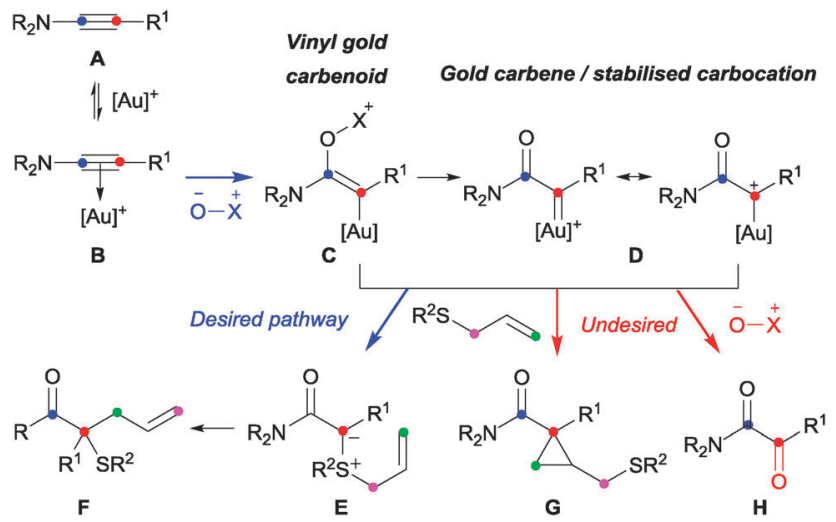

Scheme 3 A gold-catalysed intermolecular oxidation - intermolecular trapping manifold for ynamides.

activation or oxidation of the ynamide $(\mathbf{A} \rightarrow \mathbf{B} \rightarrow \mathbf{C}) \cdot{ }^{15}$ Ylide formation must then compete successfully with cyclopropanation (G) and oxidation (H) of organogold species $\mathbf{C}$ and/or $\mathbf{D}$.

After exploring a variety of parameters including catalyst, solvent and oxidant with various ynamides (see ESI $\dagger$ ), reaction conditions were found to effect the complexity-increasing cascade from ynamides 1 into the functionalised tertiary thioethers 4 (Scheme 4). Competing double-oxidation to form imido-ketones $(\mathbf{H})$ was observed throughout this study alongside incomplete consumption of $\mathbf{1}$, and this pathway was exclusively observed using $\mathrm{Au}(\mathrm{III})$ or $\mathrm{NHC}-\mathrm{Au}(\mathrm{I})$ catalysts. However, good conversion and selectivity for the formation of $\mathbf{4}$ was achieved using a combination of cationic gold phosphite complex Au-I alongside methylpicolinate $N$-oxide 3. The reactions were run in dichloromethane at room temperature and reagents $1: 2: 3$ were added at the start of the reaction in a near-stoichiometric $1: 1.2: 1.3$ ratio respectively (Scheme 4 ). Recourse to continual or portion-wise slow-addition strategies that maintain a low concentration of oxidant to favour reaction with the desired nucleophile was avoided. This preference was maintained throughout the study in order to explore the extent and influences on the observed chemoselective preference for sulfide attack at the organogold intermediate. ${ }^{13,16}$

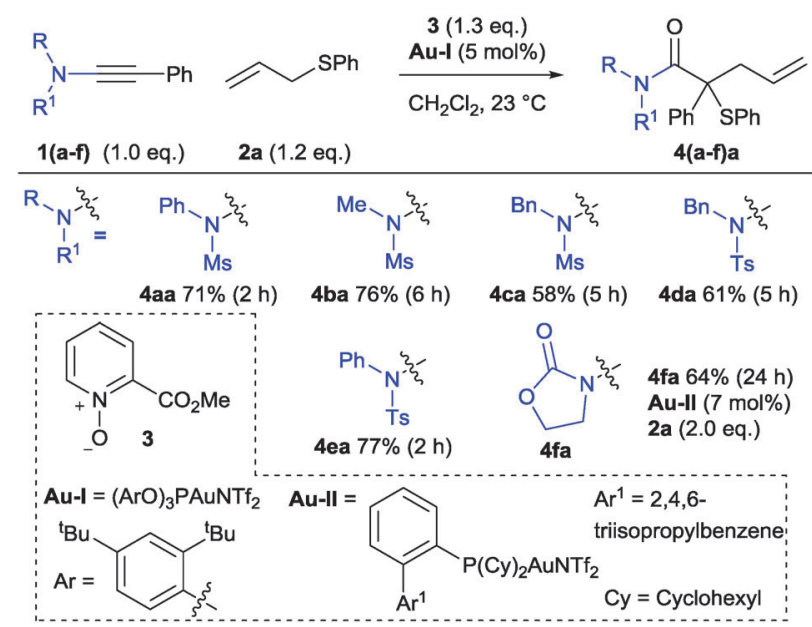

Scheme 4 The ynamide-based Doyle-Kirmse type reaction.
Ynamides bearing $N$-phenyl and $N$-methyl groups reacted similarly (4ba vs. 4aa) while an $N$-benzyl equivalent was less suited affording a lower yield of 4ca with competing overoxidation observed. ${ }^{17}$ The trend between $N$-phenyl and $N$-methyl was maintained in the $p$-tolylsulfonamide series, albeit with slightly higher yields (Scheme 4, 4da and 4ea). In both cases, products from cyclisation of $\mathbf{C} / \mathbf{D}$ onto the $N$-substituent were not observed. An ynamide prepared from imidazole was unreactive. Oxazolidinone-derived ynamide $\mathbf{1 f}$ could be used to form $\mathbf{4 f a}$ but greater conversion and selectivity for ylide formation was achieved using a bulky phosphine-derived catalyst Au-II in place of the phosphite-gold complex, identifying potentially significant influencing factors between different types of ynamides (see ESI $\dagger$ ).

The sulfonamide-derived ynamides were used throughout the remainder of this study into structural effects on the relative efficacy of the standard conditions. The reaction remained effective in the presence of $S$-aryl, $S$-benzyl- and $S$-alkyl allyl sulfides 2b-e as well as bisallylsulfide $2 \mathbf{f}$ (Scheme 5). Unsurprisingly, a more electron-deficient aryl bromide substituent renders the ylide pathway less competitive against oxidation with $\sim 30 \%$ yield of the over-oxidation product determined from the crude reaction mixture.

The role of the ynamide $C$-substituent was next tested under the standard conditions (Scheme 6). None of the desired product from ylide formation was observed when anisole-derived ynamide $\mathbf{1 g}$ was reacted with sulfides $\mathbf{2 a}$ or $\mathbf{2 b}$ with the over-oxidation product 5 instead predominating. A preference for over-oxidation against an intramolecular reaction has recently been reported when using $p$-anisole derived ynamide. ${ }^{10 f}$ In contrast, both inductively and mesomerically electron-withdrawing groups were well tolerated (4hb and 4ib). Alkyl-substituted gold carbenoids are prone to 1,2-insertion ${ }^{9 a}$ and this intramolecular pathway was indeed preferred, affording 6 with low conversion of the hexynederived ynamide $\mathbf{1 j}$. A conjugated ene-ynamide $\mathbf{1 k}$ did not follow a recently reported $4-\pi$-electrocyclisation pathway ${ }^{10 c}$ instead reacting productively to afford functionalised tertiary allylic thioether 4ka.

By analogy to the effects encountered in diazo-derived metal carbene chemistry, changing the ynamide $C$-substituent was expected to influence the reactivity of the resulting carbenoid. ${ }^{2}$ However, the impact of the electronic influence on the chemoselectivity of these ynamide-based reactions is informative: the formation of the desired compound 4 requires the active organogold species to react preferentially with the polarisable sulfur nucleophile 2 in preference to the dipolar oxygen-nucleophile 3.

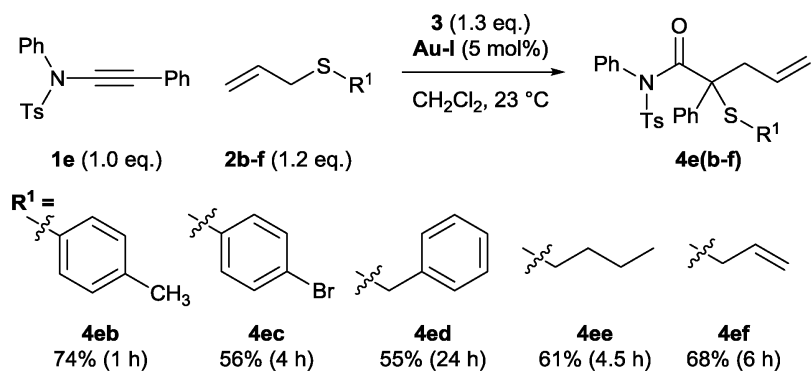

Scheme 5 Effect of the non-migrating group on the allyl sulfide. 


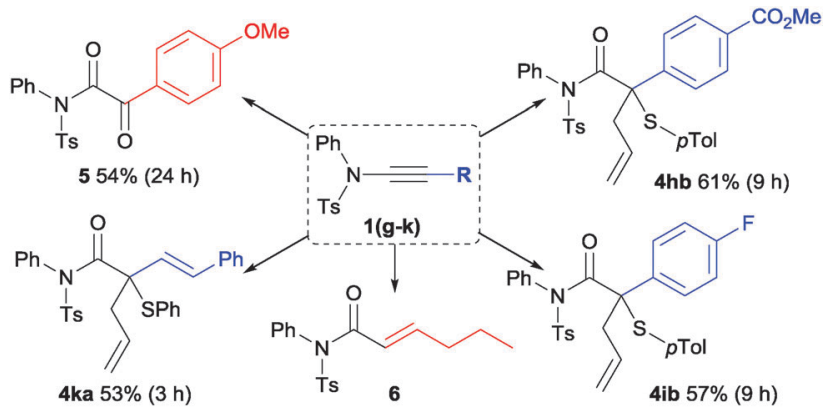

Scheme 6 Effect of the ynamide $C$-substituent on the reaction outcome.

Nucleophilic attack can occur either alongside- or after elimination of methylpicolinate (Scheme 3, $\mathbf{C} \rightarrow \mathbf{E} v$ s. $\mathbf{C} \rightarrow \mathbf{D} \rightarrow \mathbf{E}$ ) ${ }^{8-10,12,13}$ Considering the contrasting natures of the nucleophiles, a favourable attack of the sulfide on the vinyl gold carbenoid $\mathbf{C}$, bearing a relatively-uncharged carbon centre, appears more likely than on the cationic carbon of gold carbene $\mathbf{D}$. The strongly $\pi$-acidic phosphite ligand affords significant cationic character to $\mathbf{D}$ by decreasing the ability of gold to donate electron density. ${ }^{18}$ However, these same electronic characteristics should aid the desired chemoselectivity by disfavouring the elimination of methylpicolinate required to form $\mathbf{D}$. On this basis, the reactivity of electron-rich ynamide $1 \mathrm{~g}\left(\mathrm{R}^{1}=p-\mathrm{MeOC}_{6} \mathrm{H}_{4}\right)$ can be explained by the mesomeric contribution from the anisole MeO-group aiding elimination of the nucleofuge to form $\mathbf{D}$ and disfavouring ylide formation.

Sulfides with substituted allyl units were then explored to ascertain if a [2,3]-sigmatropic rearrangement was operative. Pleasingly, and in contrast with other systems, ${ }^{7}$ both 2-vinyltetrahydrothiophene $\mathbf{2 g}$ and cinnamyl derivative $2 \mathbf{h}$ underwent clean reactions. The functionalised 8-membered sulfur heterocycle 4 eg and the tertiary thioether bearing a vicinal tertiary stereogenic centre $\mathbf{4 e h}$ are the products expected from sulfur-ylide formation and [2,3]-sigmatropic rearrangement with allylic inversion (Scheme 7). $\star^{2,6,19}$ Interestingly, formation of $4 \mathbf{e h}$ proceeded with higher diastereoselectivity than is normally observed from the

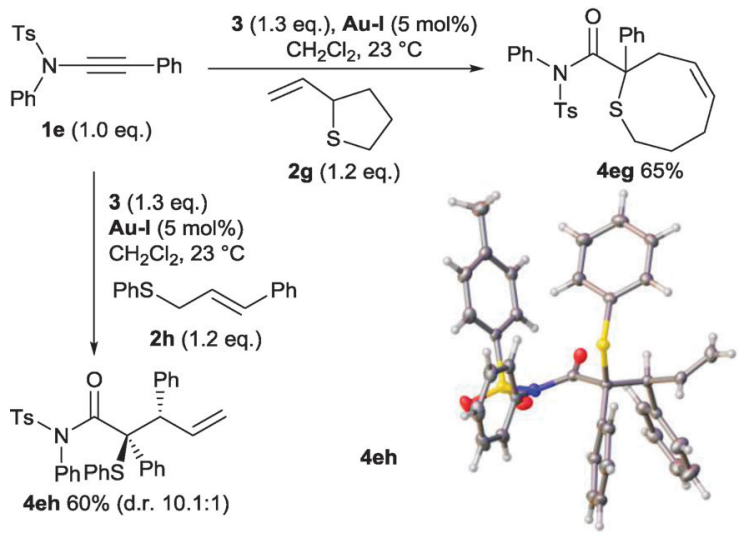

Scheme 7 Reactions of ynamide-derived sulfur ylides displaying allylic inversion. Crystal structure of $4 \mathrm{eh}$ with ellipsoids drawn at the $50 \%$ probability level. reaction of substituted allyl sulfides $2 \mathbf{h}$ with metal carbenes derived from donor-acceptor diazocompounds. ${ }^{6,19}$ The relative stereochemistry of the major diastereoisomer was confirmed by $\mathrm{X}$-ray analysis (Scheme 7) and is consistent with a preference for the cinnamyl phenyl group to be positioned anti to the amide group across an envelope transition state. Rapid access to these $N, N$-disubstituted amide derivatives by the ynamide strategy may therefore provide additional synthetic advantages.

In conclusion, a gold-catalysed cascade reaction is reported for the practical complexity-increasing synthesis of functionalised tertiary thioethers directly from ynamides. Selectivity for sulfur ylide formation over competing processes is rationalised and can be achieved when all reagents are added at the start of the process with a near-stoichiometric reagent loading. The products are consistent with those expected from [2,3]-sigmatropic rearrangement of allyl sulfonium ylides. This report demonstrates that ynamides can be used as synthetically attractive replacements to 1,1-disubstituted diazocompounds in intermolecular processes.

The authors thank the European Union FP7 Marie-Curie IEF for funding (Fellowship to MDS: ESAMY). We thank the EPSRC UK National Crystallography Service at the University of Southampton for the collection of the crystallographic data ${ }^{20}$ and Dr Louise Male (University of Birmingham) for its analysis. The facilities used in this research were part supported through Birmingham Science City AM2 by Advantage West Midlands and the European Regional Development Fund.

\section{Notes and references}

$\ddagger$ Crystal data for 4eh: $\mathrm{C}_{36} \mathrm{H}_{31} \mathrm{NO}_{3} \mathrm{~S}_{2}, M=589.74$, monoclinic, $a=$ 16.0175(3) ̊, $b=9.6290(2) \AA, c=19.5279(5) \AA, \beta=98.570(1)^{\circ}, U=$ 2978.2(1) $\AA^{3}, T=120(2) \mathrm{K}$, space group $P 2_{1} / c, Z=4,34108$ reflections measured, 6813 unique $\left(R_{\text {int }}=0.0607\right)$ which were used in all calculations. The final $R_{1}$ was $0.0458(I>2 \sigma(I))$ and $\mathrm{w} R\left(F_{2}\right)$ was 0.1150 (all data).

1 Reviews: (a) J. S. Clark, Nitrogen, Oxygen and Sulfur Ylide Chemistry: A Practical Approach in Chemistry, Oxford University Press, Oxford, 2002; (b) Sulfur Mediated Rearrangements: Topics in Current Chemistry, ed. E. Schaumann, vol. 275, Springer, Heidelberg, 2007.

2 (a) W. Kirmse and M. Kapps, Chem. Ber., 1968, 101, 994. Reviews: (b) M. P. Doyle, W. H. Tamblyn and V. Bagheri, J. Org. Chem., 1981, 46, 5094; (c) M. P. Doyle, M. A. McKervey and T. Ye, Modern Catalytic Methods for Organic Synthesis with Diazo Compounds, Wiley-Interscience, New York, 1998; (d) A.-H. Li, L.-X. Dai and V. K. Aggarwal, Chem. Rev., 1997, 97, 2341; (e) D. M. Hodgson, F. Y. T. M. Pierard and P. A. Stupple, Chem. Soc. Rev., 2001, 30, 50; for a recent example; $(f)$ M. S. Holzwarth, I. Alt and B. Plietker, Angew. Chem., Int. Ed., 2012, 51, 5351.

3 Use of tosyl hydrazone diazo-precursors: (a) V. K. Aggarwal and C. L. Winn, Acc. Chem. Res., 2004, 37, 611; (b) Y. Li, Z. Huang, X. Wu, P.-F. Xu, J. Jin, Y. Zhang and J. Wang, Tetrahedron, 2012, 68, 5234.

4 (a) P. W. Davies and S. J.-C. Albrecht, Chem. Commun., 2008, 238; (b) P. W. Davies and S. J.-C. Albrecht, Angew. Chem., Int. Ed., 2009, 48, 8372; (c) P. W. Davies, Pure Appl. Chem., 2010, 82, 1537; (d) P. W. Davies and S. J.-C. Albrecht, Synlett, 2012, 70.

5 (a) Y. Kato, K. Miki, K. F. Nishino, K. Ohe and S. Uemura, Org. Lett., 2003, 5, 2619; (b) D. Yadagiri and P. Anbarasan, Chem. - Eur. J., 2013, 19, 15115.

6 P. W. Davies, S. J.-C. Albrecht and G. Assanelli, Org. Biomol. Chem., 2009, 7, 1276.

7 Cyclopropanation not ylide formation was observed when allyl sulfides bearing more substituted alkenes were employed, see ref. $4 d$.

8 Alkynyl sulfoxide reactions: (a) N. D. Shapiro and F. D. Toste, J. Am. Chem. Soc., 2007, 129, 4160; (b) G. Li and L. Zhang, Angew. Chem., Int. Ed., 2007, 46, 5156; (c) B. Lu, Y. Li, Y. Wang, D. H. Aue, Y. Luo and L. Zhang, J. Am. Chem. Soc., 2013, 135, 8512. 
9 (a) P. W. Davies, A. Cremonesi and N. Martin, Chem. Commun., 2011, 47, 379; (b) P. W. Davies, A. Cremonesi and L. Dumitrescu, Angew. Chem., Int. Ed., 2011, 50, 8931.

10 (a) D. Vasu, H.-H. Hung, S. Bhunia, S. A. Gawade, A. Das and R.-S. Liu, Angew. Chem., Int. Ed., 2011, 50, 6911; (b) A. Mukherjee, R. B. Dater, R. Chaudhuri, S. Bhunia, S. N. Karad and R.-S. Liu, J. Am. Chem. Soc., 2011, 133, 15372; (c) S. Bhunia, C.-J. Chang and R.-S. Liu, Org. Lett., 2012, 14, 5522; (d) R. B. Dateer, K. Pati and R.-S. Liu, Chem. Commun., 2012, 48, 7200; (e) L.-Q. Yang, K.-B. Wang and C.-Y. Li, Eur. J. Org. Chem., 2013, 2775; $(f)$ K.-B. Wang, R.-Q. Ran, S.-D. Xiu and C.-Y. Li, Org. Lett., 2013, 15, 2374; $(g)$ R. Liu, G. N. Winston-McPherson, Z.-Y. Yang, X. Zhou, W. Song, I. A. Guzei, X. Xu and W. Tang, J. Am. Chem. Soc., 2013, 135, 8201; Sulfoxides: $(h)$ C.-W. Li, K. Pati, G.-Y. Lin, S. A. Sohel, H.-H. Hung and R.-S. Liu, Angew. Chem., Int. Ed., 2010, 49, 9891; (i) C.-F. Xu, M. Xu, Y.-X. Jia and C.-Y. Li, Org. Lett., 2011, 13, 1556; Nitrene addition; $(j)$ C. Li and L. Zhang, Org. Lett., 2011, 13, 1738.

11 Ynamide preparations: (a) Y. Zhang, R. P. Hsung, M. R. Tracey, K. C. M. Kurtz and E. L. Vera, Org. Lett., 2004, 6, 1151; (b) A. Coste, G. Karthikeyan, F. Couty and G. Evano, Angew. Chem., Int. Ed., 2009, 48, 4381; (c) T. Hamada, X. Ye and S. S. Stahl, J. Am. Chem. Soc., 2008, 130, 833; Reviews; (d) K. A. DeKorver, H. Li, A. G. Lohse, R. Hayashi, Z. Lu, Y. Zhang and R. P. Hsung, Chem. Rev., 2010, 110, 5064; (e) G. Evano, A. Coste and K. Jouvin, Angew. Chem., Int. Ed., 2010, 49, 2840.

12 For the first report and then representative examples from other groups: (a) L. Ye, W. He and L. Zhang, J. Am. Chem. Soc., 2010, 132, 8550; (b) L. Zhang, Acc. Chem. Res., 2014, 47, 877; (c) M. Xu,
T.-T. Ren and C.-Y. Li, Org. Lett., 2012, 14, 4902; (d) A. S. K. Hashmi, T. Wang, S. Shi and M. Rudolph, J. Org. Chem., 2012, 77, 7761; (e) D. Qian and J. Zhang, Chem. Commun., 2012, 48, 7082; (f) J. Fu, H. Shang, Z. Wang, L. Chang, W. Shao, Z. Yang and Y. Tang, Angew. Chem., Int. Ed., 2013, 52, 4198; $(g)$ S. Bhunia, S. Ghorpade, D. B. Huple and R.-S. Liu, Angew. Chem., Int. Ed., 2013, 52, 4229; (h) G. Henrion, T. E. J. Chavas, X. Le Goff and F. Gagosz, Angew. Chem., Int. Ed., 2013, 52, 6277.

13 (a) W. He, L. Xie, Y. Xu, J. Xiang and L. Zhang, Org. Biomol. Chem., 2012, 10, 3168; (b) Y. Luo, K. Ji, Y. Li and L. Zhang, J. Am. Chem. Soc., 2012, 134, 17412; (c) K. Ji, Y. Zhao and L. Zhang, Angew. Chem., Int. Ed., 2013, 52, 6508; (d) L. Xie, Z. Liang, D. Yan, W. He and J. Xiang, Synlett, 2013, 1809; (e) C. Wu, Z. Liang, D. Yan, W. He and J. Xiang, Synthesis, 2013, 2605.

14 For two-component intermolecular metathesis, see ref. $10 b$.

15 Gold carbenes with thiols: R. J. Mudd, P. C. Young, J. A. JordanHore, G. M. Rosair and A.-L. Lee, J. Org. Chem., 2012, 77, 7633.

16 Regular portionwise addition of 3 affords $81 \%$ (by ${ }^{1} \mathrm{H}$ NMR) by maintaining a low concentration of oxidant relative to the sulfide.

17 Co-elution prevented accurate yields of over-oxidised product.

18 D. Benitez, N. D. Shapiro, E. Tkatchouk, Y. Wang, W. A. Goddard III and F. D. Toste, Nat. Chem., 2009, 1, 482.

19 (a) T. Fukudat, R. Irie and T. Katsuki, Tetrahedron, 1999, 55, 649; (b) S. Kitagaki, Y. Yanamoto, H. Okubo, M. Nakajima and S. Hashimoto, Heterocycles, 2001, 54, 623; (c) Y. Nishibayashi, K. Ohe and S. Uemura, J. Chem. Soc., Chem. Commun., 1995, 1245; (d) X. Zhang, Z. Qu, Z. Ma, W. Shi, X. Jin and J. Wang, J. Org. Chem., 2002, 67, 5621.

20 S. J. Coles and P. A. Gale, Chem. Sci., 2012, 3, 683. 